\title{
LAS ESTRATEGIAS DE COMUNICACIÓN EN DOS CLUBES DE FÚTBOL DEL ECUADOR
}

\author{
THE COMMUNICATION STRATEGIES ANALYSIS IN TWO ECUADORIAN SOCCER CLUBS
}

\author{
Guillermo Zambrano ${ }^{1}$ \\ Maria Belén Zambrano Pontón ${ }^{2}$ \\ Rosario L. Haro Velastegui ${ }^{3}$
}

\section{Resumen}

El objetivo es analizar los procesos de comunicación de dos clubes de futbol ecuatoriano: el Centro Deportivo Olmedo y el Mushuc Runa Sporting Club. Se colectaron datos primarios por medio de encuestas dirigidas a públicos internos y externos. La población interna son todos los funcionarios de los clubes. La población externa son los socios tanto de los clubes como los socios de los gremios de periodistas de las provincias de Chimborazo y Tungurahua (Ecuador). Los resultados evidencian una falta de estrategia de comunicación. Se concluye que los clubes han realizado acciones aisladas pero sin planificación. Por último se proponen unos lineamientos para un plan estratégico de comunicación.

\section{Palabras clave}

Comunicación interna, comunicación externa, estrategias de comunicación, plan estratégico.

\section{Abstract}

The objective is to analyze the communication processes of the Ecuadorian clubs: the Olmedo Sports Center and the Mushuc Runa Sporting Club. Primary data were collected through surveys aimed at internal and external audiences. The internal population all the officials of the clubs. The external population is the partners of both the clubs and the partners of the governments of the provinces of Chimborazo and Tungurahua (Ecuador). The results show a lack of communication strategy. It is concluded that the clubs have carried out isolated actions without planning. Finally, they propose guidelines for a strategic communication plan.

\section{Keywords}

Internal communication, external communication, communication strategies, strategic plan.

\footnotetext{
${ }^{1}$ Universidad Nacional de Chimborazo (Ecuador). Correo electrónico: gzambrano@unach.edu.ec

${ }^{2}$ Universidad Federal de Viçosa (Brasil). Correo electrónico: belen.zambrano.p@hotmail.com.

${ }^{3}$ Universidad Técnica de Ambato (Ecuador). Correo electrónico: charyharo@yahoo.es.
} 


\section{Introducción}

La comunicación es indispensable para el normal desarrollo de las actividades dentro de una organización. En las instituciones, la estrategia de comunicación cumple un papel importante. Una manera correcta de informar al público sobre las acciones que están siendo realizadas, puede llegar a ser un instrumento para alcanzar las metas y los objetivos propuestos. Por ejemplo, los dirigentes o gerentes deportivos cuentan con distintas estrategias empresariales para gestionar la organización, en las cuales, el aspecto comunicacional se constituye una importante herramienta que permite mantener en contacto a la institución con los clientes internos y externos de los clubes, mejorando la imagen y haciéndola más competitiva. Como destaca Molina (2010), es indispensable la comunicación para que se desarrollen los valores y las estrategias de una organización. Es importante la manera en que son divulgadas y adoptadas para que las empresas definan claramente su identidad.

Al aplicar estrategias de comunicación, lo primero es definir el mensaje, partiendo de la identidad de la organización, destacando su misión y su visión. Después se planifica y se pone en práctica lo propuesto, definiendo con claridad a qué se dedica la organización y los servicios que ofrece.

Actualmente, la comunicación se desarrolla principalmente de manera digital en un mundo cada vez más conectado. Según Oliveira (2018), este tipo de comunicación acerca a organizaciones y públicos, estrechando los lazos que son determinantes para el éxito de las estrategias de la organización. El marketing permite en este sentido, que los consumidores (públicos) estén más y mejor informados, sintiéndose involucrados con las organizaciones (Reis et al., 2016).

La manera como las organizaciones deciden comunicarse con sus públicos, constituye la estrategia de comunicación. $\mathrm{Su}$ diseño implica que los objetivos de comunicación parten de políticas establecidas por las organizaciones. Su importancia radica en el empeño de colaboración para lograr los objetivos de la organización. De este modo, la comunicación busca establecer lazos fuertes y duraderos entre las organizaciones y sus públicos. Uno de los mayores desafíos para los profesionales de la comunicación y el marketing es lograr que estas actividades logren fidelizar a los públicos (Limeira, 2010).
Con base en lo expuesto, el problema de investigación es indagar cómo se desarrolla y comunica la información en los clubes de fútbol de la serie A de la región central del Ecuador. El objetivo es analizar los procesos de comunicación de los clubes del centro del país ecuatoriano; específicamente, identificar las percepciones y expectativas de los públicos internos y externos de estos clubes, respecto a la comunicación y proponer estrategias de comunicación. Para el efecto, fueron escogidos los clubes Centro Deportivo Olmedo (CDO), representante de la ciudad de Riobamba-Ecuador y el Mushuc Runa Sporting Club (MRSC), que representa a la ciudad de Ambato-Ecuador. En la época de la colecta de los datos los dos equipos se encontraban en la serie A del campeonato ecuatoriano de futbol.

\section{La comunicación estratégica}

Martínez y Nosnik (1988) definen la comunicación como: «el proceso por medio del cual una persona se pone en contacto con otra a través de un mensaje, y espera que esta última dé una respuesta, sea una opinión, actitud o conducta» (p. 12). La comunicación consiste en enviar mensajes, ideas y opiniones entre las personas; sea de manera hablada, escrita, gesticular, etc. Así, para describir el proceso de comunicación se emplea un modelo de comunicación, que incluye seis elementos: emisor, mensaje, receptor, canal, retroalimentación y ruido (Biagi, 2009).

Según Biagi (2009) el emisor es la fuente o el lugar donde se origina el mensaje, el receptor es el destino del mensaje, el canal es el medio por el que se transmite, el ruido es cualquier distorsión que interfiere con una comunicación clara y la retroalimentación ocurre cuando el receptor procesa el mensaje y envía de regreso una respuesta al emisor.

Al definir la comunicación organizacional, Fernández (1997) señala que es el intercambio de mensajes entre los miembros de una organización, sea entre sí y/o con el ambiente interno y externo a la institución, añadiendo que el flujo de comunicación en las organizaciones son todas las técnicas utilizadas para influenciar a los públicos internos y externos, sus opiniones o determinadas conductas con la finalidad de que la organización alcance los objetivos propuestos. La comunicación organizacional es parte del fortalecimiento y desarrollo institucional, que se basa en las acciones comunicativas que parten de los vínculos de la organización y se proyectan potenciando la 
visibilidad de la misma y de sus objetivos.

Los públicos son quienes reciben el mensaje. Así, Xifra (2003) establece tres variables que determinan la manera cómo reciben las informaciones del emisor; esas variables son generadas a través del reconocimiento del problema, buscando información y procesándola; reconociendo las restricciones, es decir, sí tienen pocas posibilidades de elección en una situación, la información tiene poco valor para ellos; y, reconocimiento de la implicación cognitiva. Esta variable permitirá distinguir sí la conducta comunicativa del público será activa o pasiva.

En ese sentido, la comunicación puede ser interna y externa. La interna es considerada como el intercambio de mensajes, datos e información entre el talento humano y los distintos niveles jerárquicos dentro de la organización. Trelles (2002) considera que la comunicación interna centra su interés en los procesos y subprocesos procurando que los participantes de la organización ejecuten las acciones planeadas en busca de cumplir los objetivos propuestos y la manera como son viabilizados los canales de información. Manning (1992) considera que sin los canales de comuni $\neg$ cación, el mensaje institucional no llegaría a los públicos destino.

Así, para Pizzolante (2004), la comunicación interna define situaciones en donde dos o más personas intercambian o comparten principios e ideas de la empresa con visión global. Por su parte, Chiavenato (1996) considera que el clima laboral constituye el medio interno de una organización, que involucra diferentes aspectos, como el tipo de organización, la tecnología, las políticas, las metas operacionales, los reglamentos internos, las actitudes, los sistemas de valores y los factores sociales. Es importante considerar que la percepción de cada colaborador es distinta, porque los individuos son diferentes, determinando el comportamiento de la organización, por lo que el clima laboral también es diferente de una entidad a otra.

García (1998) sostiene que la comunicación interna se enfoca en las acciones y metodologías, planificando los recursos para el desarrollo de la organización. Los recursos de la comunicación interna son: apoyo decidido de la alta dirección, donde se toman las decisiones estratégicas de la comunicación interna; profesionalización de sus funciones y decisiones para una capacitación completa y técnica sobre liderazgo organizacional; recursos económicos; promover los espacios de participación, retroalimentación y opinión del personal de trabajo.
La comunicación interna cuenta con cuatro aspectos básicos: información (normas de acuerdo a objetivos establecidos); difusión (captar, persuadir y convencer a la gente); formación y socialización (fomentar la cultura organizacional a través de distintas actividades como: capacitaciones, celebraciones y acciones de recreación); y, participación (promoviendo la colaboración del equipo de trabajo).

Figura 1. Aspectos básicos de comunicación interna.

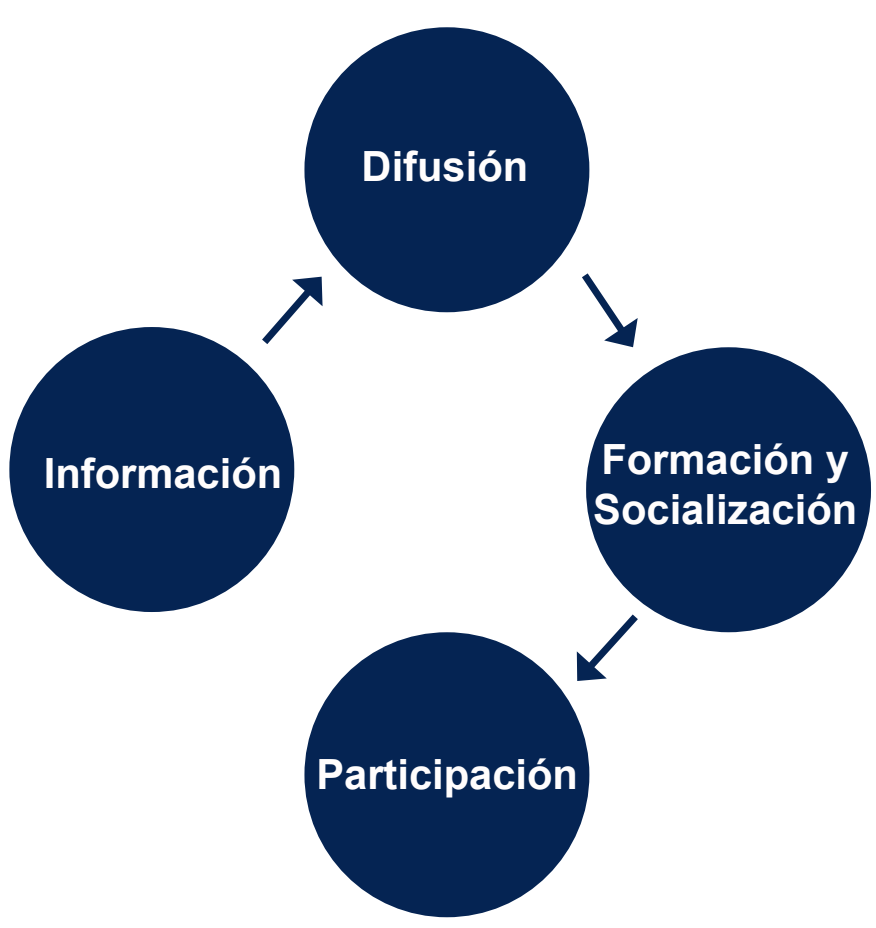

Fuente: elaboración propia.

Existen diferentes tipos de comunicación interna. Según Judge et al. (2009), la comunicación descendente, generalmente es utilizada para informar las metas planteadas, otorgar las instrucciones de las tareas a desempeñar, entre otros. Así, la comunicación hacia abajo es ejercida desde un nivel superior de la organización para con los subalternos y la comunicación para arriba fluye hacia un nivel superior en la organización utilizada para proporcionar retro-alimentación a los de arriba, informarles sobre el progreso de las metas y darles a conocer problemas actuales (Judge et al., 2009, p. 354).

Continuando con la clasificación, la comunicación lateral según Judge et al. (2009), sucede cuando la comunicación es entre los miembros del mismo grupo de trabajo, al mismo nivel, entre los gerentes del mismo 
rango. «Este tipo de medio utiliza más herramientas como las comunicaciones directas verbales, reuniones de grupos informales, uso de los correos electrónicos y retroalimentaciones en reuniones de grupos» (Judge et al., 2009, p. 355).

Otra forma de comunicación es la externa, siendo considerada como el enlace entre la organización y los públicos externos (PE) a ella, pudiendo ser: clientes, proveedores, medios u otras instituciones. Los $\mathrm{PE}$ están compuestos por personas que forman parte del entorno de la organización y así como influyen, también son influenciados. De ese modo, en el caso de la comunicación externa, se evidenció que desde la teoría se pueden encontrar sistematizaciones, como la ofrecida por Muriel y Rota (1980) quienes, de acuerdo al nivel de relación que mantienen los públicos externos con la institución, los clasifican en generales y específicos.

Según estos autores, los públicos externos generales se hacen presentes a través de la opinión pública y desde los determinados públicos de una organización, es decir de los medios de comunicación colectiva que conectan con la colectividad. Los específicos, son aquellos que se encuentran relacionados directamente con la institución y su clasificación se establece a partir de los motivos de contacto de estos con la empresa. Las autoras separan en cinco tipos: clientes, proveedores, localidad, otras instituciones públicas y los medios de comunicación (Muriel y Rota, 1980).

La necesidad de comunicar, de transmitir hacia el exterior, debe realizarse de manera efectiva y sobre todo planificada. Hernández (2002), destaca algunos aspectos para elaborar un plan de comunicación, entre los que constan: los objetivos del plan, establecer la política, análisis de la situación actual; establecer: misión, visión y valores; estrategias; cronograma de actividades; presupuesto; ejecución; control y monitoreo. Asimismo, Hodge, Anthony y Lawrence (2003) consideran que no es posible establecer una única y correcta forma de estructurar las organizaciones, pues es algo que depende de su propia realidad, de cada contexto, del ambiente en el cual se desarrolla la actividad, así como de los objetivos, metas y de la cultura de cada club.

Por otro lado, los canales de comunicación que las organizaciones suelen utilizar para comunicarse con sus públicos pueden ser clasificados en: canales mediatizados y canales directos, siendo los primeros los que requieren algún tipo de tecnología, utilizando algún vehículo físico externo. Estos se dividen en: canales impresos y electrónicos. Ejemplo de los impresos, serían: los boletines de prensa, periódicos, revistas, carteleras, etc. Por su parte, los electrónicos se dividen en: canales de gran alcance y los que se dirigen de persona a persona, dentro de los de gran alcance están: televisión, radio, Internet, y los dirigidos de persona a persona, sirven para comunicarse con personas o sistemas sociales y tienen la posibilidad de retroalimentación más rápida.

Los canales electrónicos de gran alcance llegan a una gran parte de la población y en la actualidad. Las nuevas tecnologías de comunicación e información han invadido nuestra vida diaria, han borrado las fronteras y conectan inmediatamente a la mayoría de las sociedades del mundo, permitiendo una interacción casi inmediata. Estos medios son una representación cultural de la época en que vivimos, son un producto humano que va a permanecer y a los cuales debemos adaptarnos de una manera racional, crítica y objetiva. Dentro de este tipo de canales están: las páginas web y las redes sociales (Leoni, 2009).

Así, los canales directos son los que no requieren ninguna clase de tecnología, existiendo un contacto directo entre el emisor y el receptor. Algunos de los principales medios directos con que puede contar la institución son: entrevistas, conferencias, juntas, reuniones y cualquier tipo de comunicación cara a cara.

Por lo tanto, la comunicación con los públicos internos y externos se sugiere sea de manera coordinada. La comunicación con los socios y aficionados debe darse a través de la elaboración de las herramientas que permitan mantenerlos informados, con noticias actualizadas y rápidas, permitiendo también obtener una retroalimentación, por medio de sugerencias en beneficio del club (Gómez y Opazo, 2007).

De acuerdo con Véliz (2014) la comunicación contribuye de manera estratégica en los procesos organizacionales, es decir, la manera de relacionar la participación, integración y coordinación de los funcionarios. En la percepción de Laguna y Mesa (2010) la estrategia constituye el camino para lograr el éxito, ayuda a las organizaciones a superar los constantes desafíos y consideran que cuando la comunicación fluye de manera apropiada es porque fueron utilizados correctamente los principios.

La construcción de las estrategias surge a partir de la comunicación, otorgando nuevas alternativas de 
interacción social, de los procesos de comunicación con los públicos internos y externos, con la finalidad de que todas las decisiones de la organización estén desarrolladas de una manera totalmente estratégica, existiendo una interacción entre lo que se planifica, lo que se comunica y la manera como es interpretado por los públicos (Arcila, 2014).

Una planificación estratégica de comunicación se debe iniciar por identificar los públicos de interés para la organización, eso va a depender de la cultura corporativa, los objetivos organizacionales y comunicacionales; luego definir las estrategias, elaborar el tipo de mensajes y los canales de comunicación a utilizar, imaginar la reacción de los públicos al mensaje y así lograr el impacto deseado en el área de comunicación (Córdoba, 2011).

La comunicación estratégica en las organizaciones, de acuerdo con Da Costa (2005) tiene un obstáculo al ser vinculada con los sistemas de gestión, pues si no tienen una cultura preparada para una administración estratégica pueden dejar de lado la comunicación de sus prioridades.

Scheinsohn (2004) considera que la comunicación estratégica agrupa las actitudes, aptitudes y conocimientos que son aplicados por los funcionarios de las organizaciones. El autor considera que, ese proceso selecciona los discursos que deben estar integrados entre la organización y los públicos. La comunicación estratégica en las organizaciones representa una táctica gerencial indispensable dentro de las empresas, promoviendo y fortaleciendo el desempeño laboral, habilidades comunicacionales, garantizando la efectividad y eficiencia de los mensajes que se transmiten (Paz, Paz, \& Kadi, 2017).

Generalmente, las organizaciones que han triunfado son las que han enfocado de mejor manera su estrategia, aplicando una lógica diferente, la innovación. Abriendo un espacio nuevo y desconocido en el mercado (Mauborgne, 2013). De ese modo, la estrategia describe en teoría cómo se va a alcanzar un objetivo, ofreciendo líneas directrices y direccionamientos para el programa global. Según Wilcox, Cameron y Xifra (2006), se puede destacar una estrategia general, o bien el programa puede tener diversas estrategias, en función de los objetivos y los públicos seleccionados.

\section{Metodología}

Para esta investigación, el diseño muestral utilizado fue no probabilístico por conveniencia. Y fue así que, por su localización geográfica, por las facilidades de accesibilidad y financieras para la colecta de los datos fueron escogidos los dos clubes del centro del país ecuatoriano que se encuentren en similares condiciones ya que en la época de la colecta de los datos (2014), los dos equipos se encontraban en la serie A del campeonato ecuatoriano de futbol: el centro Deportivo Olmedo (CDO) y el Mushuc Runa Sporting Club (MRSC).

\section{Caracterización de los Clubes en estudio}

El CDO, el club más antiguo del Ecuador, fundado el 11 de noviembre de 1919. Durante estos casi cien años de permanencia en el futbol ecuatoriano se ha convertido en la identidad de los riobambeños. En 1994, regresó a la serie A, desde ahí se empoderó en el «Ciclón» para seis años después, coronarse por primera vez campeón del fútbol ecuatoriano en el año 2000, aunque actualmente se encuentra en la serie B del campeonato ecuatoriano de futbol.

Por su parte, MRSC se fundó en el año 2002, participando primero en ligas barriales de las parroquias Juan Benigno Vela y Pilahuín, en la provincia de Tungurahua. Cinco años tuvieron que pasar para que el gerente del club, Luis Alfonso Chango, y un grupo de 130 socios, formen el equipo de fútbol profesional, participando en el torneo de tercera categoría, logrando el año siguiente (2008), el ascenso a la segunda división, del campeonato organizado por la Asociación de Fútbol de Tungurahua.

En el año 2011, en el tercer intento, el equipo ascendió a la serie B. Su mayor éxito lo consiguieron en el año 2013, cuando ascendieron a la serie A, acompañando al club Olmedo, en calidad de vice campeones. Actualmente, también se encuentra en la serie B del campeonato ecuatoriano de futbol. Fueron escogidos esos dos equipos de futbol por la accesibilidad para la colecta de los datos, así como la historia y trayectoria antigua y reciente en el futbol ecuatoriano. 


\section{Población y muestra}

Para fines de esta investigación, la población fue dividida en dos partes: la población total de públicos internos y la población de públicos externos, siendo el total de la población de públicos internos 95 personas, de los dos clubes en estudio, como se presenta en la Tabla 1.

Tabla 1. Población públicos internos de los Clubes en estudio para la colecta de datos.

\begin{tabular}{|c|c|c|c|}
\hline \multicolumn{2}{|c|}{ Centro Deportivo Olmedo: } & \multicolumn{2}{c|}{ Club Deportivo Mushuc Runa: } \\
\hline Detalle & Cantidad & Detalle & Cantidad \\
\hline Cuerpo técnico & 8 & Cuerpo técnico & 27 \\
\hline Jugadores & 28 & Jugadores & 8 \\
\hline Personal administrativo & 9 & Personal administrativo & 4 \\
\hline Trabajadores & 5 & Trabajadores & 45 \\
\hline Total: & $\mathbf{5 0}$ & Total: & 45 \\
\hline
\end{tabular}

Fuente: elaboración propia.

Cabe resaltar que dado el tamaño de la población correspondiente al público interno (funcionarios) de los dos clubes en estudio, se optó por trabajar con el $100 \%$ de esa población. Y solo en el caso de la población de los públicos externos fue necesario determinar el tamaño de la muestra, tomando en cuenta el número total de la población de públicos externos (ver Tabla 2).

Tabla 2. Determinación de la muestra de los públicos externos de los clubes en estudio.

\begin{tabular}{|l|c|c|}
\hline \multicolumn{2}{|c|}{ Socios y periodismo deportivo } \\
\hline Detalle & Cantidad & Porcentaje \\
\hline Socios Centro Deportivo Olmedo & 1233 & $84 \%$ \\
Socios Mushuc Runa Sporting Club & 150 & $10 \%$ \\
Círculo de Periodistas Deportivos de Chimborazo & 56 & $4 \%$ \\
Asociación de Periodistas Deportivos de Tungurahua & 35 & $2 \%$ \\
\hline Total Público Externo: & 1474 & $100 \%$ \\
\hline Muestra Públicos Externos: & 305 & \\
\hline
\end{tabular}

Fuente: elaboración propia. 
De ese modo, la manera como fue determinada la muestra de 305 encuestas a los públicos externos fue a través de la fórmula de muestreo de poblaciones finitas, donde: $\mathrm{N}$ es el total de la población; Za es el valor de la distribución corresponde a 1.96; $\mathrm{p}(0,05)$ es la proporción esperada; $\mathrm{q}$ es 1-p (1-0,05) es $0,95 \mathrm{y} \mathrm{d}$ es el nivel de confianza en este caso $(95 \%)$

$$
n=\frac{N^{*} Z_{a}^{2} p^{*} q}{d^{2} *(N-1)+Z_{a}^{2} * p^{*} q}
$$

\section{Técnica y colecta de datos}

Para llevar a cabo este trabajo se utilizaron datos primarios y la técnica escogida para la colecta de los datos fue la encuesta, con la finalidad de analizar la manera como son utilizadas las estrategias, acciones y herramientas comunicativas en los clubes en estudio. Las encuestas fueron realizadas a mediados de la temporada 2014 del campeonato ecuatoriano de futbol de la serie A. Se realizaron dos encuestas diferentes, una dirigida a los públicos internos y la otra a los externos, la primera encuesta al público interno que laboraba en los clubes Centro Deportivo Olmedo y Mushuc Runa Sporting Club, incluía jugadores, cuerpo técnico, personal administrativo y trabajadores.
Esa encuesta fue elaborada sobre cuatro aspectos básicos de la comunicación interna: información (normas de acuerdo a objetivos establecidos); difusión (captar, persuadir y convencer a la gente); formación y socialización (fomentar la cultura organizacional a través de distintas actividades como: capacitaciones, celebraciones y acciones de recreación); y, participación (promoviendo colaboración del equipo de trabajo).

La encuesta dirigida a los públicos externos fue realizada a los socios de los clubes Centro Deportivo Olmedo y Mushuc Runa Sporting Club; a los periodistas agremiados al Círculo de Periodistas Deportivos de Chimborazo y a la Asociación de Periodistas Deportivos de Tungurahua.

\section{Resultados y Discusión}

La comunicación en los clubes deportivos: el caso del Centro Deportivo Olmedo y el Mushuc Runa Sporting Club

Para comprender como es la comunicación al interior de los equipos de futbol del centro del país, se preguntó a los públicos internos de estas organizaciones la manera como son informados sobre las acciones de su club, como se puede apreciar en la Tabla 3.

Tabla 3. Información al interior del club

\begin{tabular}{|c|c|c|}
\hline Alternativas: & Frecuencia: & $\%$ \\
\hline $\begin{array}{l}\text { Correo Electrónico } \\
\text { Intranet } \\
\text { Cartelera } \\
\text { Equipo de trabajo } \\
\text { Medios de Comunicación }\end{array}$ & $\begin{array}{c}6 \\
14 \\
15 \\
57 \\
3\end{array}$ & $\begin{array}{c}6 \% \\
15 \% \\
16 \% \\
60 \% \\
3 \%\end{array}$ \\
\hline Total & 95 & 100 \\
\hline
\end{tabular}

Fuente: elaboración propia.

Se evidencia que la comunicación ocurre, mayoritariamente, de manera lateral, pues la misma fluye entre los miembros del mismo grupo de trabajo, al mismo nivel. Utilizando instrumentos de comunicación verbales, correos electrónicos, de alguna manera un tanto más informal, estando en este grupo los equipos de trabajo y los correos electrónicos. Así la cartelera, el intranet y los medios de comunicación constituirían en este caso una información descendente, ya que el fluye de arriba abajo.
Con relación a la pregunta sobre el clima de apertura y confianza al interior del club, fue evidenciado que la mayoría (59\%) de los encuestados, respondieron que no existe un clima de apertura y confianza al interior del club y el $41 \%$ dice lo contrario, notando que los públicos internos de los clubes deportivos del centro del país ecuatoriano, sienten falta de un mejor clima de relacionamiento entre los directivos y el personal.

A continuación, fue consultado si son tomadas en cuenta 
las ideas y sugerencias de los públicos internos de la organización por parte de los directivos de los clubes, el $43 \%$ de los entrevistados al interior del respectivo club, afirman que son bienvenidas las opiniones, ideas y sugerencias, mientras que el 57\% manifiesta lo contrario. Evidenciándose que necesita ser trabajada la retroalimentación de ideas y sugerencias de los públicos internos, eso con el propósito de continuamente mejorar.

En ese sentido, Emery y Trist (1965) y Katz y Kahn (1987) consideran que la comunicación interna en las organizaciones, debería contar con la retroalimentación entre los diferentes actores; solo así se podrá controlar y evaluar las acciones que están siendo realizadas, así como la importancia que las nuevas ideas tienen con la intención de mejorar los aspectos dentro de esas organizaciones.

Con relación a la capacitación, fue posible identificar que el $61 \%$ de los encuestados manifiestan que son aplicadas estrategias de capacitación para mejorar el desempeño del talento humano en los Clubes Deportivos de Fútbol del Centro del país y el 39\% sostiene lo contrario. Resaltando ese resultado, ya que representa el intento de los clubes deportivos en aplicar estrategias y mantener capacitados a sus públicos internos.

En la interrogante sobre la evaluación de los canales de comunicación utilizados por el club deportivo al cual pertenecen los encuestados, el $60 \%$ sostiene que los canales de comunicación interno de la institución están entre regulares y malos, contra, el $40 \%$ que sí cumplen con las expectativas estando entre bueno, muy bueno y excelente. Infiriéndose que, según la percepción de los públicos internos de cada club en estudio, deben mejorar los canales de comunicación utilizados para informar, como presentada en la Figura 2.

Figura 2. Evaluación canales internos de comunicación

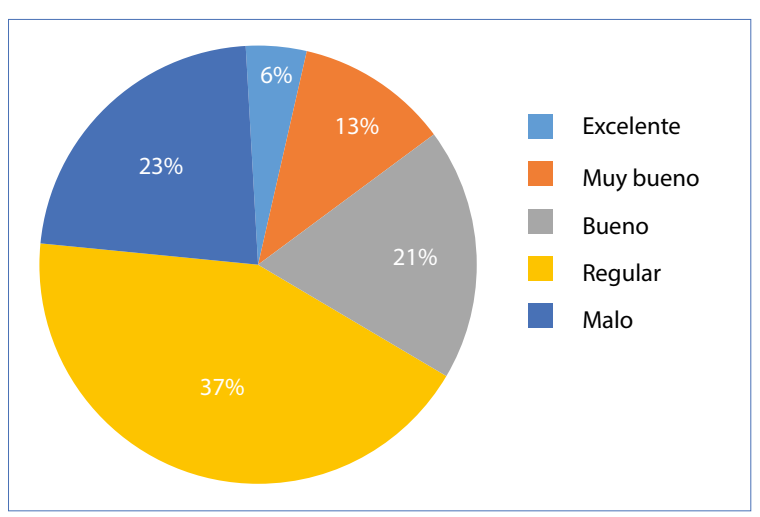

Fuente: elaboración propia.
A continuación, son presentadas las percepciones de los públicos externos, de acuerdo con Sánchez (2009) antiguamente, los clubes de fútbol se relacionaban principalmente, con los medios de comunicación, ya que ellos transmitían su identidad corporativa y su gestión empresarial. Así, actualmente, con las nuevas tecnologías de la información y comunicación, vienen tomando mayor protagonismo los socios, aficionados, directivos, y otras instituciones públicas y privadas para cumplir también con esa función.

De esa manera, los públicos externos que fueron escogidos en esta investigación fueron los socios de los clubes de la región central del país y los periodistas deportivos, para de esta manera conocer el nivel de satisfacción sobre la información proveniente de las organizaciones en estudio. El 92\% de los encuestados afirman que conocen sobre la existencia de una página web de los clubes de la serie A de la región central del país y el $8 \%$ manifiesta que no conocen o que no tienen.

Las tecnologías de la información invadieron el espacio de la comunicación, modificando la relación entre las organizaciones y los públicos. Antiguamente, el poder era ejercido por los grandes medios de comunicación, siendo realizado casi exclusivamente por ellos; ahora esa información se encuentra disponible, accesible y puede ser comunicada también por los públicos, eso es posible a través de las redes sociales (Terra, 2011).

Infiriendo que, aunque los clubes dispongan de una página web, los encuestados aseguran que la información disponible en dichos portales no es suficiente en un 90\% y sólo $10 \%$ consideran que la información es suficiente. Evidenciándose que, las organizaciones deportivas deben realizar la actualización de las informaciones que se encuentran disponibles en dichos portales web, pues solo así se va a logar tener informados a los públicos externos de las acciones del club.

En ese sentido, para Garcia-Gómez (2001) deben ser presentadas de manera clara las ideas y los propios contenidos compartidos en las páginas web para cualquier tipo de organización. Inclusive para el autor, algunas veces son presentados un arte y diseño llamativos, restando importancia a la innovación y a los contenidos, acrecentando también, que deben ser actualizadas de manera frecuente las informaciones disponibles en esos portales, lo que incentivará que los públicos visiten las páginas web con cierta periodicidad, porque saben que ahí pueden obtener informaciones veraces y actualizadas 
de lo que es de su interés.

Así, para De Amorim (2014), la comunicación digital constituye un potencial que no ha sido muy bien explotado por las organizaciones deportivas, sugiriendo que deben ser estudiados los públicos, con la finalidad de entender, que es lo que desean de informaciones y cuáles son los contenidos relevantes para ellos y así, mejorar de forma continua esa información. Acrecentando, que existe poco uso de los medios digitales, siendo que son la herramienta del presente y del futuro.

Otra manera, que todavía persiste para comunicar las informaciones a los públicos externos son los boletines de prensa, que son documentos enviados a los diferentes medios de comunicación, con el propósito de informar, comunicar e invitar a los diferentes eventos que son realizados dentro del club. Así, el 96\% de encuestados sostiene que son enviados boletines informativos, sea de manera física o digital a los medios de comunicación, socios e hinchada, mientras que el $4 \%$ considera que no.

Aunque sean enviados boletines de prensa, la mayoría de los encuestados $83 \%$ consideran que no son claras las informaciones que contienen esos documentos enviados por los clubes analizados del centro del país y $17 \%$ consideran que son claros y precisos en las informaciones que contienen, resaltando nuevamente la importancia de la claridad de las informaciones que son comunicadas a los públicos.

La pregunta que consultó sobre las convocatorias para Rueda de Prensa por parte de los clubes del centro del país analizados, muestran que el $90 \%$ de los encuestados respondieron que son convocados a ruedas de prensa por parte de los clubes y solo $10 \%$ que no. Resaltando la importancia de mantener un canal de comunicación efectivo entre los clubes deportivos y los medios de comunicación, pues ellos constituyen el nexo entre las organizaciones y los públicos externos.

Con relación al conocimiento de la existencia de un departamento o unidad de comunicación en los clubes, $87 \%$ de los encuestados manifiesta que los clubes cuentan con una unidad o departamento de comunicación y el $13 \%$ dice lo contrario.

Así, aunque exista un departamento de comunicación, el desempeño del personal que labora en ese departamento, para el $84 \%$ de los encuestados consideran que está entre regular y malo y el $16 \%$ lo evalúa entre bueno, muy bueno y excelente, como evidenciada en la Figura 3.

Figura 3. Evaluación canales internos de comunicación

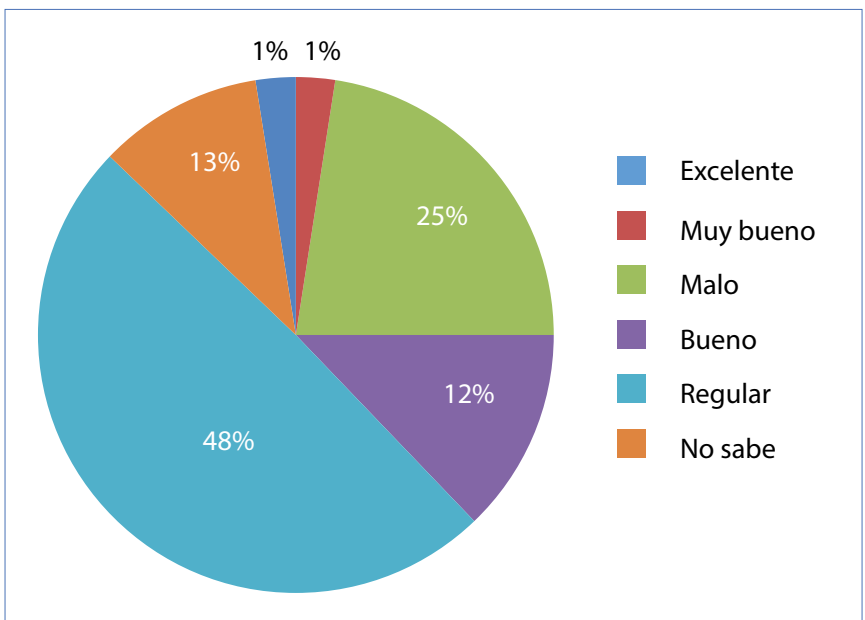

Fuente: elaboración propia.

En ese sentido, Da Costa (2005) concluye que las organizaciones no siempre disponen de personal capacitado para la planificación estratégica y la comunicación, así como, generalmente, no son realizadas investigaciones que permitan identificar el interés de sus públicos ni tampoco tienen metodologías definidas para medir sus resultados de comunicación.

\section{Propuesta para la construcción de las estrategias de comunicación de los clubes deportivos: Centro Deportivo Olmedo y Mushuc Runa Sporting Club}

Con esta investigación fue posible evidenciar que la comunicación en las organizaciones deportivas, se viene desarrollando de manera simple y básica, identificando que los clubes analizados no contaban (a la fecha de la colecta de los datos) con estrategias comunicacionales, situación que ha obligado a realizar campañas aisladas, carentes de planificación.

Siendo necesario que las organizaciones socialicen: misión, visión y valores, para que el talento humano se empodere de fines y objetivos comunes; la difusión de las actividades debe ser clara y precisa, para evitar confusiones, buscando captar, persuadir y convencer a sus públicos. Así también las páginas web deben tener contenidos interesantes y actualizados, para vincularse de manera efectiva con sus públicos externos. Así, en este 
artículo se utilizó para la construcción de las estrategias de comunicación para los clubes deportivos, el modelo basado en los elementos para la construcción de un plan de comunicación propuesto por Hernández (2002), Figura 4. Evaluación canales internos de comunicación representada en la Figura 4.

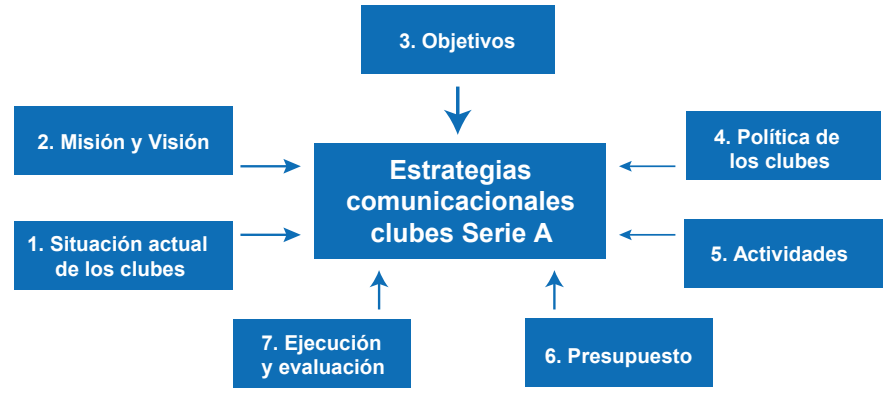

Fuente: Adaptado de Hernández (2002).

De esta manera se elaboró primero un diagnóstico de la situación actual del club, seguido de la elaboración de la misión y visión del club deportivo, los objetivos generales y específicos que se proponen alcanzar. De la misma manera es importante determinar cuál es la política de los clubes, las acciones o actividades planeadas para alcanzar los objetivos y metas, así como, el presupuesto con el que cuenta el club, la ejecución y evaluación del desarrollo de todo lo planificado.

\section{Diagnóstico de la situación actual de los clubes}

En el modelo de gestión del Centro Deportivo Olmedo, no se ha ponderado la necesidad de tener una comunicación profesional. Por lo tanto, no existe una unidad, dirección o departamento de comunicación institucional, que planifique, ejecute y evalúe las estrategias para posicionar tanto en los públicos internos, como externos la marca institucional. Como acciones aisladas se han generado medios como un portal web y cuentas en redes sociales Facebook y Twitter, cuyos contenidos necesitan mayor trabajo periodístico. Los medios de comunicación social validan vocerías que no siempre responden a las políticas de sus dirigentes. Gestando así un mensaje confuso que muchas veces se malinterpreta. Si bien existe una identidad en la ciudad de Riobamba, a nivel nacional el club no la ha construido ni ha fidelizado públicos.

En cambio, el equipo Mushuc Runa sí tiene un relacionista público, aunque la entidad carece de un plan de comunicación, funcionando con estrategias digitales como: página web y redes sociales.
En ese sentido, como resultado del diagnóstico de los clubes en estudio, fueron definidas las Fortalezas, Oportunidades, Debilidades y Amenazas (FODA) institucional de los equipos en estudio (Tabla 4).

Tabla 4. FODA Institucional Centro Deportivo Olmedo y Mushuc Runa Sporting Club.

\begin{tabular}{|c|c|}
\hline Fortalezas & Oportunidades \\
\hline $\begin{array}{l}\text { - Empoderamiento de la } \\
\text { hinchada } \\
\text { - } \text { Representatividad de } \\
\text { ciudad y del sector } \\
\text { indígena } \\
\text { - La vocería que generan } \\
\text { los equipos }\end{array}$ & $\begin{array}{l}\text { - Crear nuevas hinchadas } \\
\text { - Expectativa de la región } \\
\text { - Establecer alianzas que } \\
\text { mejoren los canales de } \\
\text { comunicación }\end{array}$ \\
\hline Debilidades & Amenazas \\
\hline $\begin{array}{l}\text { - Falta de cultura } \\
\text { organizacional } \\
\text { - La forma de relacionarse } \\
\text { con sus hinchadas y } \\
\text { demás públicos externos } \\
\text { - Una marca débil }\end{array}$ & $\begin{array}{l}\text { - Consolidación de otros } \\
\text { equipos en el territorio } \\
\text { nacional } \\
\text { - Poca cobertura } \\
\text { periodística de medios } \\
\text { nacionales } \\
\text { - Ausencia de hinchas en el } \\
\text { estadio }\end{array}$ \\
\hline
\end{tabular}

Fuente: elaboración propia

Misión: consolidar los procesos y canales de comunicación de los clubes Centro Deportivo Olmedo y Mushuc Runa para mejorar los contenidos que se emiten a socios, hinchadas, periodismo y aficionados en general.

Visión: al finalizar el 2020, Olmedo y Mushuc Runa serán en la región central del país, el referente de imagen deportiva donde se hace posible lo soñado y comenzará a reflejarse en el país.

Objetivo General: mejorar los contenidos informativos de los clubes Centro Deportivo Olmedo y Mushuc Runa Sporting Club, como equipos profesionales, diversos, convergentes, utilizando estrategias $\mathrm{y}$ herramientas comunicacionales.

Objetivos Específicos: aplicar estrategias de comunicación de los clubes Centro Deportivo Olmedo y Mushuc Runa Sporting Club; Difundir las actividades que cumplen los clubes a través de boletines de prensa elaborados de manera clara, precisa y concisa; Mejorar las interrelaciones con los periodistas deportivos del país; generar herramientas Web 2.0 . 
Para fines de esta investigación las herramientas de la Web 2.0 están relacionada con las estrategias de divulgación de los contenidos, sean: videos, audios, fotos, imágenes y links, para que sean utilizados en direccionar las acciones estratégicamente con la finalidad de tener una interacción con los públicos.

Políticas: El diálogo permanente de los dirigentes con los funcionarios, cuerpo técnico y deportistas de los clubes Centro Deportivo Olmedo y Mushuc Runa; Establecer una política de puertas abiertas, con los periodistas, los medios y la hinchada, entregando información profesional que proyecte la imagen integral de la institución.

Estrategias: Fortalecer la Comunicación institucional, con profesionales experimentados que establezcan las estrategias y las acciones que permitan que los clubes Centro Deportivo Olmedo y Mushuc Runa, se consoliden en el imaginario colectivo; Difusión masiva y alternativa; enfocando el trabajo diario de los equipos Olmedo y Mushuc Runa, sus productos y servicios, mediante el uso de medios tradicionales y alternativos; Establecer nuevos diálogos y canales con los periodistas deportivos del país; Diseñar carteleras institucionales en las que se destaque la misión y la visión de los clubes (Tabla 5).

Tabla 5. Estrategias de Comunicación y actividades propuestas.

\begin{tabular}{|l|l|}
\hline \multicolumn{1}{|c|}{ Estrategias } & \multicolumn{1}{|c|}{ Actividades } \\
\hline $\begin{array}{l}\text { Fortalecer la } \\
\text { comunicación } \\
\text { institucional }\end{array}$ & $\begin{array}{l}\text { Estudio de factibilidad. } \\
\text { Adecuaciones del espacio físico. } \\
\text { Selección y yumanontos. } \\
\text { humanoción del talento } \\
\text { Fortalecimiento del área de comunicación. } \\
\text { Evaluación. }\end{array}$ \\
\hline $\begin{array}{l}\text { Ajustes administrativos y técnicos. } \\
\text { masiva y } \\
\text { alternativa }\end{array}$ & $\begin{array}{l}\text { Estudio de mercado. } \\
\text { Diseño gráfico. } \\
\text { Implementar comunicación web 2.0. }\end{array}$ \\
\hline $\begin{array}{l}\text { Nuevos } \\
\text { diálogos y } \\
\text { canales con } \\
\text { los periodistas } \\
\text { deportivos }\end{array}$ & $\begin{array}{l}\text { Realizar y actualizar la cartografía de } \\
\text { periodistas deportivos del país. } \\
\text { Establecer canales de comunicación para } \\
\text { entregar productos comunicacionales. } \\
\text { Rueda de prensa. }\end{array}$ \\
\hline $\begin{array}{l}\text { Diseñar } \\
\text { carteleras } \\
\text { institucionales } \\
\text { internas }\end{array}$ & $\begin{array}{l}\text { Diseñar una cartelera en la que se } \\
\text { abarquen temas institucionales y de interés } \\
\text { destacándose misión, visión de los clubes. }\end{array}$ \\
\hline
\end{tabular}

Fuente: elaboración propia.
Después de la ejecución de las estrategias, se elaboró un presupuesto básico con los costos de las actividades programadas en un primer momento, pudiendo ser adaptado según la fecha de la ejecución, como presentada en la Tabla 6.

Tabla 6. Presupuesto individual (para cada club) de las actividades propuestas.

\begin{tabular}{|c|c|c|c|}
\hline Actividad & Periodicidad & Cantidad & Costo \\
\hline Estudio de factibilidad & Una vez & 1 & 1000 \\
Adecuaciones & Una vez & 1 & 2000 \\
Equipamiento & Una vez & 1 & 3000 \\
Contratación de talento & Mensual & 1 & 12000 \\
humano & Una vez & 1 & 0 \\
Implementación & Una vez & 1 & 0 \\
Evaluación & Semanal & 48 & 0 \\
$\begin{array}{c}\text { Elaboración cartelera } \\
\text { interna }\end{array}$ & Semanal & 450 & 0 \\
$\begin{array}{c}\text { Diseño gráfico } \\
\text { Vinculares a medios } \\
\text { alternativos }\end{array}$ & Semanal & 160 & 0 \\
$\begin{array}{c}\text { Realizar y organizar la } \\
\text { cartografía }\end{array}$ & Semestral & 2 & 0 \\
$\begin{array}{c}\text { Establecer canales de } \\
\text { comunicación }\end{array}$ & Permanente & 360 & 0 \\
\hline \multicolumn{2}{|c|}{ TOTAL } & 18000 \\
\hline
\end{tabular}

Fuente: elaboración propia.

Para dar seguimiento, fue necesario establecer un calendario planificado de las actividades realizadas, de todo lo desarrollado; fue realizada la primera evaluación, para determinar si las acciones que fueron llevadas a cabo, tuvieron los resultados planificados, de no ser así, en esta etapa es posible tomar los correctivos necesarios, encaminando nuevamente hacia la consecución de los objetivos propuestos, como se presenta en la Tabla 7.

Tabla 7. Evaluación de las actividades propuestas

\begin{tabular}{|c|c|c|c|}
\hline \multirow{2}{*}{ ACTIVIDADES } & \multicolumn{2}{|c|}{ INDICADORES } & \multirow{2}{*}{$\%$} \\
\hline \multirow{2}{*}{ Carteleras internas } & Elaborados & Vistos & \multirow{2}{*}{50} \\
\cline { 2 - 3 } & 48 & 24 & \\
\cline { 1 - 3 } Ruedines de prensa & Elaborados & Publicados & \multirow{2}{*}{20} \\
\cline { 2 - 3 } & 60 & 12 & \\
\hline \multirow{2}{*}{ Páginas web } & Invitados & Asistentes & \multirow{2}{*}{32} \\
\cline { 2 - 3 } & 25 & 8 & \multirow{2}{*}{10} \\
\cline { 2 - 3 } Redes sociales & 1000 & 100 & \\
\cline { 2 - 3 } & Visitas & "Me gusta" & \multirow{2}{*}{16,66} \\
\hline
\end{tabular}

Fuente: elaboración propia. 
Como queda evidenciado, después de la primera evaluación, fue necesario que los clubes tomen algunas medidas para mejorar los contenidos principalmente, de los boletines de prensa, con el propósito de que sea publicada una mayor cantidad de información referente a las organizaciones deportivas. Así también debían mejorar los canales de comunicación con los periodistas, para incentivar que asistan a las ruedas de prensa convocadas por los clubes, en mayor número y así divulgar las novedades de los equipos, promocionar las páginas web y las redes sociales para conseguir más visitas de los aficionados.

\section{Consideraciones finales}

La comunicación en el Centro Deportivo Olmedo ha sido realizada sin planificación, ya que, no existía (a la fecha de la colecta de los datos) una unidad, dirección o departamento específico, de comunicación institucional, que planifique, ejecute y evalúe las estrategias para posicionar tanto en los públicos internos como externos la marca institucional.

En dicho club, se vienen realizando acciones aisladas, generando medios como un portal web y cuentas en redes sociales Facebook y Twitter, cuyos contenidos se evidencian con una necesitad de mayor trabajo periodístico. Los medios de comunicación social validan vocerías que no siempre responden a las políticas de sus dirigentes. En ese sentido, puede ser generado un mensaje un tanto confuso y muchas veces hasta malinterpretado por los públicos.

Por su parte, la comunicación del equipo Mushuc Runa Sporting Club, a pesar de contar con un relacionista público, quien ejecuta y dirige las acciones de comunicación, la entidad carece de un plan de comunicación, funcionando únicamente con estrategias digitales como: página web y redes sociales.

Una fortaleza con la que cuentan los dos equipos de fútbol profesional, es su sentido de identidad y pertenencia en la ciudad de Riobamba y Ambato, respectivamente, pero la realidad a nivel regional y nacional es muy diferente. Estos clubes no han construido ni han fidelizado públicos, constituyendo una oportunidad de expansión de su hinchada en el ámbito regional y nacional y, es ahí que radica la importancia de las estrategias de comunicación.

La propuesta del modelo teórico propuesto por Hernández
(2002) y que fue utilizado en la práctica en este artículo para la elaboración de las estrategias comunicacionales en los clubes deportivos, se presenta como un modelo que podría auxiliar en la construcción de cualquier tipo de estrategias, en cualquier organización, no solo en el ámbito deportivo. Este modelo permite identificar si los objetivos están siendo cumplidos o si necesitan ser replanteados con el propósito de mejorar la comunicación.

Una contribución en este trabajo es el debate generado a partir de la manera como viene desarrollándose, en la práctica, la comunicación en los clubes de pequeño tamaño del fútbol ecuatoriano y una limitación de este trabajo es que podrían haber sido analizados otros clubes de fútbol en el país, aunque ese tema por el momento no fue el foco de esta investigación.

Quedan así, abiertas las posibilidades para nuevas investigaciones en busca de la ampliación del conocimiento en el área de la comunicación y de las estrategias en las organizaciones, ya que nuevos estudios pueden acompañar las acciones de los dirigentes. Además sería importante que se analicen otros clubes del balompié ecuatoriano, trayendo a análisis, otros equipos internacionales, con características similares para así comparar las estrategias comunicacionales que están siendo desarrolladas por los clubes deportivos, tanto nacional como internacionalmente.

\section{Referencias bibliográficas}

1. Arcila C. M. (2014). Comunicación estratégica, y estrategias de comunicación para el desarrollo de las organizaciones (tesis de pregrado). Universidad Católica de Pereira (Colombia). Recuperado de http://hdl.handle.net/10785/2367.

2. Biagi, S. (2009). Impacto de los medios de comunicación. México: Cengage Learning.

3. Chiavenato, I (1996). Administración de Recursos Humanos. (3a ed.). México: Mc Graw Hill.

4. Córdoba, S. O. (2011). La Comunicación en la gestión de la Responsabilidad Social Empresarial. Correspondencias \& análisis, (1), 137-156.

5. Da Costa B. W. (2005). A comunicação empresarial estratégica: definindo os contornos de um conceito. Conexão-Comunicação e Cultura, 4(7), 11-20.

6. De Amorim, R. R. (2014). Comportamento do 
Consumidor Esportivo no Ambiente Virtual: Um Estudo das Ações e Práticas de Mercado Brasileiro, Espanhol e Português. Podium: Sport, Leisure and Tourism Review, 3(2), 68-84. doi:10.5585/podium. v3i2.92

7. Emery, F., Trist, E. L. (1965). The causal texture of organizational environments. Human Relations, 18(1), 21-32. doi: https://doi. org/10.1177/001872676501800103

8. Fernández, C. (1997). La Comunicación en las Organizaciones. México: Trillas.

9. García, J. J. (1998). La Comunicación Interna. Madrid: Ediciones Díaz de Santos.

10. García-Gómez, J. C. (2001). Portales de internet: concepto, tipología básica y desarrollo. El profesional de la información, 10(7-8), 4-13.

11. Gómez, S. y Opazo, M. (2007). Características estructurales de un club de fútbol profesional de élite. IESE Business School - Universidad de Navarra. Documento de Investigación No. 705. Barcelona España.

12. Hernández, A. J. (2002). Planificar la comunicación. Revista Latina de Comunicación Social, 5(48). Recuperado de: http://www.ull.es/publicaciones/ latina/2002/latina48marzo/4812ajhernandez.htm

13. Hodge, B. J., Anthony W. P. \& Lawrence M. G. (2003). Teoría de la organización: Un enfoque estratégico. (6a ed.) Madrid: Pearson Prentice Hall.

14. Judge, T., Robbins, S. P., y Sobral, F. (2009). Comportamiento organizacional. Estados Unidos: Pearson.

15. Katz, D., \& Khan, R. L. (1987). Psicologia Social das Organizações. (3a ed.) São Paulo: Atlas.

16. Bermúdez, F. A., Ramírez, I., y Mesa, A. (2010). Estrategia de Comunicación para fortalecer la imagen organizacional de Copextel S.A. división territorial Las Tunas. Observatorio de la Economía Latinoamericana, (140).

17. Leoni, H. S. L. (2009). Los medios de comunicación y las nuevas tecnologías de la Información y Comunicación: ¿Inocentes o Culpables?, En Contribuciones a las Ciencias Sociales, febrero 2009. Recuperado de www.eumed.net/rev/cccss/03/ sl5.htm

18. Limeira, T. M. V. (2003). Administração das Comunicações de Marketing. En: Dias, S. R. (Org.). Gestão de Marketing. 1ed.São Paulo: Editora Saraiva, v. 1, p. 271-307.

19. Manning, P. K (1992). Organizational Comunication. Nueva York: Walterde Gruyter, Inc.

20. Martínez, V. de, A., \& Nosnik, A. (1988). Comunicación organizacional práctica: manual gerencial. México: Trillas.

21. Mauborgne, R. (2013). La Estrategia del Océano Azul. Bogotá: Buena Semilla.

22. Molina, V. (2010). Imagen Corporativa. (2a ed.) Quito: Hermo Grafic.

23. Muriel, M. L. y Rota, G. (1980). Comunicación institucional. Quito: Editora Andina.

24. Oliveira, D. A. D. (2018). Marketing e meios digitais: uma plataforma geradora de oportunidades de negócio para organizações esportivas (Monografia). Universidade Federal da Fronteira Sul, Cerro Largo, rio Grande do Sul, Brasil. Recuperado de https:// rd.uffs.edu.br/bitstream/prefix/1614/1/OLIVEIRA. pdf

25. Paz, J., Paz, J., y El kadi, O. (2017). Comunicación estratégica en su visión gerencial, una herramienta para lograr la autotomía caudal. Redhecs, 1(2), 5479.

26. Pizzolante, I. (2004). El poder de la comunicación estratégica. Bogotá: Editorial Pontificia Universidad Javeriana.

27. Reis, A., Iacovelo, M., Almeida, L., \& Costa Filho, B. (2016). Marketing de Relacionamento: Agregando Valor ao Negócio com Big Data. Revista Brasileira de Marketing - ReMark, 15(4), 512-523.

28. Sánchez, F. O. (2009). La comunicación no convencional en los clubes de fútbol. Pensar la Publicidad. 3(1), 121-137.

29. Scheinsohn, D. (2004). Comunicación Estratégica, Management y Fundamento de Imagen corporativa. Argentina: Ediciones Macchi.

30. Terra, C. F. (2011). O que as organizações precisam fazer para serem bem vistas nas mídias sociais 
sob a ótica da comunicação organizacional e das relações públicas. En V Congresso Científico de Comunicação Organizacional e Relações Públicas Abrapcorp, São Paulo, Brasil.

31. Trelles, I. (2002). Bases teórico-metodológicas para una propuesta de modelo de gestión de comunicación en organizaciones. La Habana: Universidad de la Habana.

32. Véliz, F. (2014). Comunicación Organizacional: Nuevas preguntas para nuevos desafíos. Quito: Editorial Quipus, CIESPAL.

33. Wilcox, D. L., Cameron G. T., \& Xifra, J. (2006). Relaciones públicas: Estrategias y tácticas. España: Editorial Pearson.

34. Xifra, J. (2003). Teoría y Estructura de las Relaciones Públicas. Madrid: Editorial Mc-Graw-Hill. 